\section{ATTRACTION OF IMMUNE CELLS BY HEAD AND NECK CANCER CELL LINES AND PRIMARY TUMOR- CONDITIONED SUPERNATANTS}

Tara Muijlwijk*, Naomi Remkes, Jos Poell, René Leemans, Ruud Brakenhoff, Rieneke van de Ven. Cancer Center Amsterdam, Amsterdam UMC, Amsterdam, Netherlands

Background Head and neck squamous cell carcinomas (HNSCC) are classified in human papillomavirus (HPV)-positive and HPV-negative tumors. In general, HPV-negative HNSCC are genetically characterized by many chromosomal gains and losses. ${ }^{1}$ Previously, we and others identified a HPVnegative subgroup with few or absent copy number alterations (CNA-silent), and a more favorable prognosis. ${ }^{2} 3$ Tumors with low copy number changes have generally been associated with high immune infiltration scores, ${ }^{4}$ but for CNA-silent versus CNA-high HPV-negative HNSCC such data are lacking.In this study we aim to unravel by functional assays immunological differences between HPV-negative and HPV-positive HNSCC, as well as between CNA-silent and CNA-high HPV-negative HNSCC. We analyzed the immune cell subsets attracted by HNSCC cell lines and by tumor-conditioned supernatants.

Methods Eight HNSCC cell lines (3 HPV-positive, 3 HPV-negative CNA-high, 2 HPV-negative CNA-silent) and 24-hour supernatants of thirteen HNSCC biopsies were used to characterize their ability to attract immune cells in a transwell migration system. A chemokine mixture was used as a positive control, while medium alone was used to determine spontaneous migration. Peripheral blood mononuclear cells (PBMCs) of various healthy donors were plated in the upper compartment and after six hours the transwell migration was quantified by flow cytometry.

Results Most HNSCC cell lines induced migration of monocytes, B cells and CD4+ T-cells up to maximal 12\%, whereas $\mathrm{CD} 8+\mathrm{T}$-cells and conventional dendritic cells (cDCs) were not attracted, irrespective of the donor. Notably, one HPVnegative CNA-silent cell line induced significantly more migration compared to the negative control and other cell lines. Tumor-conditioned supernatants promoted immune cell migration with no apparent differences between tumor sites or HPV-status. Remarkably, up to $31 \%$ of monocytes migrated to these supernatants, $9 \mathrm{x}$ more than the chemokine control. Also cDC migration was induced, whereas lymphocytes were not attracted.

Conclusions HNSCC cell lines induced monocyte, B-lymphocyte and CD4+ T-lymphocyte migration, whereas tumor-conditioned supernatants attracted monocytes and cDCs only. No difference in immune cell attraction between HPV-positive and -negative HNSCC was observed. Interestingly, one HPV-negative CNA-silent cell line induced robust immune cell migration. Currently we perform a comprehensive chemokine analysis to explain the observed migration. The noted lack of CD8 + T-cell attraction may explain why current treatments with PD-1 inhibitors are effective in only a minority of HNSCC patients. Our data could provide a means to identify patients who might most likely respond to immune checkpoint blockade and to find clues to improve CD8+ T-cell attraction.

\section{REFERENCES}

1. Leemans CR, Snijders PJF, Brakenhoff RH. The molecular landscape of head and neck cancer. Nat Rev Cancer 2018;18:269-82.

2. Smeets SJ, Brakenhoff RH, Ylstra $B$, van Wieringen WN, van de Wiel MA, Leemans CR, et al. Genetic classification of oral and oropharyngeal carcinomas identifies subgroups with a different prognosis. Cell Oncol 2009;31:291-300.

3. Cancer Genome Atlas N. Comprehensive genomic characterization of head and neck squamous cell carcinomas. Nature 2015;517:576-82.
4. Davoli T, Uno H, Wooten EC, Elledge SJ. Tumor aneuploidy correlates with markers of immune evasion and with reduced response to immunotherapy. Science 2017;355.

Ethics Approval Written informed consent was obtained from all patients from whom fresh tumor biopsies were used for research, as part of the $\mathrm{HNcol}$ protocol at the Department of Otolaryngology|Head and Neck Surgery of Amsterdam UMC (VUmc) as approved by the Institutional Review Board (2008.071|A2016.035). Buffy coats, with written consent from the donors, were purchased from the Dutch blood bank (Sanquin) and used to isolate PBMC.

http://dx.doi.org/10.1136/jitc-2021-SITC2021.935 